\title{
CHOOSING THE SUBREGIONS IN THREE-LEVEL FROSCH PRECONDITIONERS
}

\author{
Alexander Heinlein ${ }^{1}$, Oliver Rheinbach ${ }^{2,3}$ and Friederike Röver ${ }^{2}$ \\ ${ }^{1}$ Institute of Applied Analysis and Numerical Simulation, Universität Stuttgart, \\ Pfaffenwaldring 57, 70569 Stuttgart, Germany. \\ E-mail: Alexander.Heinlein@ians.uni-stuttgart.de \\ ${ }^{2}$ Institut für Numerische Mathematik und Optimierung, Technische Universität Bergakademie Freiberg \\ Akademiestr. 6, 09599 Freiberg, Germany. \\ ${ }^{3}$ Universitätsrechenzentrum (URZ), Technische Universität Bergakademie Freiberg \\ Akademiestr. 6, 09599 Freiberg, Germany.
}

Key words: Domain Decomposition, Parallel Computing, Overlapping Schwarz, Mesh Partitioning, Coarse Operator, Preconditioners, Trilinos

\begin{abstract}
Different graph partitioning methods, i.e., linear partioning, parallel hypergraph (PHG) partioning, and two approaches using ParMETIS, are considered to generate an unstructured decomposition of the second-level coarse operator of three-level FROSch (Fast and Robust Overlapping Schwarz) preconditioners in the Trilinos software library. In our context, the parallel hypergraph method shows the most consistent results.
\end{abstract}

\section{Three-Level FROSch Preconditioners}

FROSch (Fast and Robust Overlapping Schwarz) [7] is a parallel implementation of the Schwarz framework and is part of the software library Trilinos [1]. It provides parallel implementations of the GDSW (Generalized Dryja-Smith-Widlund) [5, 4] and RGDSW (Reduced-dimension GDSW) [6] overlapping Schwarz preconditioners, which make use of exotic coarse spaces. We consider the extension of these methods to three-level (R)GDSW preconditioners $[9,10]$, which arise from the recursive application of the two-level methods. They are therefore based on a hierarchy of decompositions of the original domain $\Omega$; the domain is decomposed into non-overlapping subregions $\left\{\Omega_{i 0}\right\}_{i=1, \ldots, N_{0}}$, which are further decomposed into non-overlapping subdomains $\left\{\Omega_{i}\right\}_{i=1, \ldots, N}$. Here, in order to build the subdomain hierarchy, we proceed in reverse order: We first partition $\Omega$ into the non-overlapping subdomains. Then, we build the dual graph of this decomposition based on the connectivity and obtain the subregions by partitioning this graph. The non-overlapping subdomains are then extended by layers of elements, resulting in overlapping subdomains $\left\{\Omega_{i}^{\prime}\right\}_{i=1, \ldots, N}$ with overlap $\delta$, and the subregions are extended by layers of subdomains to obtain the overlapping subregions $\left\{\Omega_{i 0}^{\prime}\right\}_{i=1, \ldots, N_{0}}$ with overlap $\Delta$. 
The three-level (R)GDSW overlapping Schwarz preconditioners are then of the form

$$
M_{\mathrm{GDSW}-3 \mathrm{~L}}^{-1}=\Phi(\overbrace{\Phi_{0} K_{00}^{-1} \Phi_{0}^{T}}^{\text {Third Level }}+\overbrace{\sum_{i=1}^{N_{0}} R_{i 0}^{T} K_{i 0}^{-1} R_{i 0}}^{\text {Second Level }}) \Phi^{T}+\overbrace{\sum_{j=1}^{N} R_{j}^{T} K_{j}^{-1} R_{j}}^{\text {First Level }},
$$

where the restriction operators $R_{i}$ to the overlapping subdomains and the respective matrices $K_{i}=R_{i} K R_{i}^{T}$ as well as the (R)GDSW basis functions $\Phi$ and the matrix $K_{0}=\Phi^{T} K \Phi$ are defined as in the two-level methods; see, e.g., [4]. Furthermore, restriction operators $R_{i 0}: V^{0} \rightarrow V_{i}^{0}:=V^{0}\left(\Omega_{i 0}^{\prime}\right)$, corresponding to the overlapping subregions $\Omega_{i 0}^{\prime}$, as well as coarse basis functions $\Phi_{0}$, spanning the (R)GDSW coarse space $V_{00}$ on the third level, are needed to build the three-level preconditioners; see $[9,10,8]$ for more details. Note that the three-level (R)GDSW approach is related to three-level or multilevel BDDC methods $[16,14,2,15]$. Our undertaking is, software-wise, of course related to other important scalable implementations of Schwarz methods, e.g., [12].

\section{Implementation}

\section{The three-level extension of GDSW type preconditioners has recently been added to the FROSch frame-} work; see also [8]. The implementation is based on the Trilinos package Xpetra, which provides a lightweight interface to the parallel linear algebra packages Epetra and Tpetra. Here, we only use the newer Tpetra-based software stack. For the assembly of the system matrix, we employ the trilinear finite element implementation on a structured domain decomposition into rectangular subdomains from the

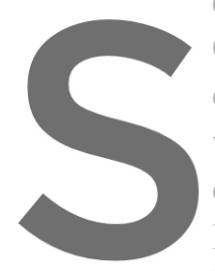

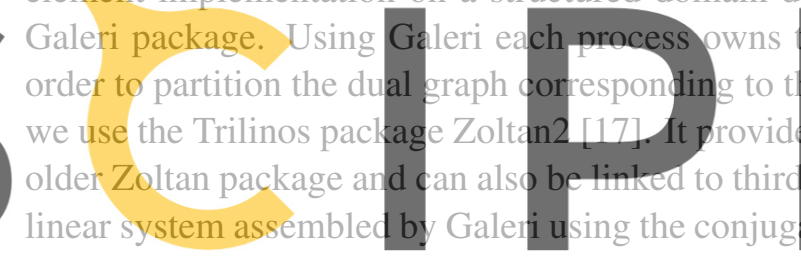
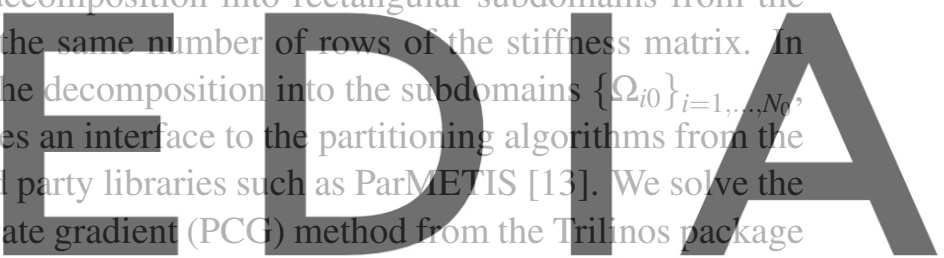

Belos, preconditioned by FROSch. Both the iterative solver and the three-level FROSch preconditioner

Register for cralled usine the ynified solver interface Stratimikos, and we iterate until we reach relative tolerance of 10 . Alf tests were performed on the compute Cluster of the Fakultat fur Mathematik und Informatik

of the TU Freiberg. We use one subdomain for each MPI rank, and one MPI rank for each processor core. We use the INTEL compiler 2020.0 version 3.1.4. To solve the arising subproblems, we always use the PardisoMKL [3] sparse direct solver.

\section{Results}

In this section, we will investigate numerically how different graph partitioning algorithms employed to construct the nonoverlapping subregions $\left\{\Omega_{i 0}^{\prime}\right\}_{i=1, \ldots, N_{0}}$ influence the performance of three-level FROSch preconditioners. As a model problem, we consider linear elasticity with homogeneous Dirichlet boundary conditions on the unit square $[0,1]^{2}$ or unit cube $[0,1]^{3}$, respectively. To build the subregions, different partitioning methods are compared: linear partitioning, which is denoted block-wise partitioning in Zoltan (Block), the parallel hyper graph (PHG) partitioner from Zoltan, and two partitioning approaches based on ParMETIS. In particular, for ParMETIS, we use either partitioning from scratch $(P)$ or repartitioning $(R)$ of the initial partition. Here, the initial partition is the linear partition. We used ParMETIS Version 4.0.3. We present results for the GDSW as well as for the RGDSW coarse space, where, for the 

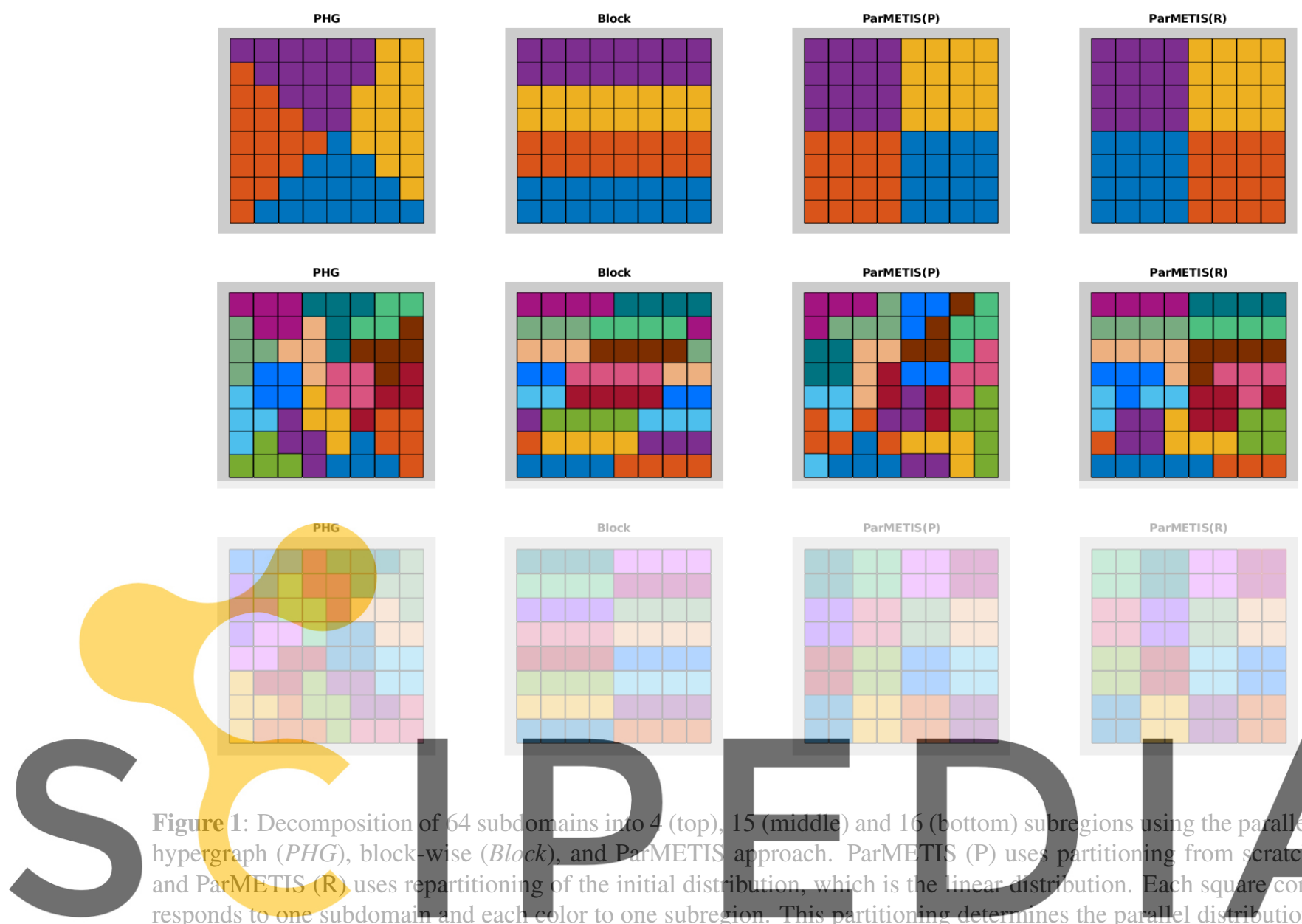

ParMetis(P)

ParMETIS(R)

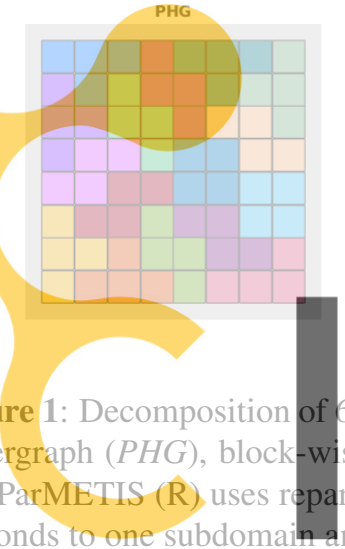

of the second-level coarse operator $K_{0}$ in FROSch and r

Register for free at https//www.scipedia.com to download the version without the watermark

RGDSW coarse space, we always consider Option I from [6,11], which is completely algebraic. Note

that, since the subregions are built based on the dual graph of the domain decomposition, the subregions are identical for the same configuration independent of the choice of the coarse space. However, the size of the GDSW and RGDSW coarse spaces may still differ significantly. In order to investigate the influence of the different partitioning algorithms on the three-level preconditioners, we focus on two aspects. First, we investigate how the convergence, i.e., the number of iterations and the estimated condition number, depend on the partitioning. Second, we will discuss the dimension of the coarse space on the third level $V_{00}$ and thus the size of the coarse matrix $K_{00}$; cf. (1). In order to do so, we consider a structured domain decomposition into 64 or 512 subdomains for two or three dimensions, respectively, and vary the 
number of subregions.

\begin{tabular}{|c|c|c|c|c|c|c|c|c|c|}
\hline & & \multicolumn{4}{|c|}{ Three-level GDSW preconditioner } & \multicolumn{4}{|c|}{ Three-level RGDSW preconditioner } \\
\hline & & PHG & Block & ParMETIS $(\mathrm{P})$ & ParMETIS(R) & PHG & Block & \begin{tabular}{|l} 
ParMETIS(P) \\
\end{tabular} & $\operatorname{ParMETIS}(\mathrm{R})$ \\
\hline \multicolumn{10}{|c|}{ 2D; \#subdomains = 64} \\
\hline \#subreg. & & \multicolumn{4}{|c|}{$\operatorname{dim}\left(K_{0}\right)=483$} & \multicolumn{4}{|c|}{$\operatorname{dim}\left(K_{0}\right)=147$} \\
\hline \multirow{4}{*}{4} & iter & 35 & 33 & 34 & 32 & 35 & 33 & 44 & 37 \\
\hline & $\kappa\left(M^{-1} K\right)$ & 19.7 & 24.8 & 19.6 & 17.6 & 17.5 & 15.1 & 30.7 & 21.7 \\
\hline & $\operatorname{dim}\left(K_{00}\right)$ & 21 & 9 & 15 & 15 & 6 & 9 & 3 & 3 \\
\hline & $\operatorname{dim}\left(K_{00}\right) / \#$ regs & 5.3 & 2.3 & 3.8 & 3.8 & 1.5 & 2.3 & 0.8 & 0.8 \\
\hline \multirow{4}{*}{15} & & 36 & 40 & 44 & 40 & 43 & 43 & 54 & 43 \\
\hline & $\kappa\left(M^{-1} K\right)$ & 21.9 & 32.5 & 30.8 & 29.2 & 26.5 & 26.3 & 43.1 & 25.8 \\
\hline & $\operatorname{dim}\left(K_{00}\right)$ & 147 & 129 & 144 & 132 & 54 & 30 & 45 & 45 \\
\hline & $\operatorname{dim}\left(K_{00}\right) / \#$ regs & 9.8 & 8.6 & 9.6 & 8.8 & 3.6 & 2.0 & 3.0 & 3.0 \\
\hline \multirow{4}{*}{16} & iter & 36 & 40 & 39 & 32 & 43 & 44 & 50 & 40 \\
\hline & $\kappa\left(M^{-1} K\right)$ & 21.4 & 33.1 & 24.8 & 21.2 & 26.5 & 27.1 & 40.3 & 24.8 \\
\hline & $\operatorname{dim}\left(K_{00}\right)$ & 147 & 87 & 99 & 99 & 51 & 21 & 27 & 27 \\
\hline & $\operatorname{dim}\left(K_{00}\right) / \#$ regs & 9.2 & 5.4 & 6.2 & 6.2 & 3.2 & 1.3 & 1.7 & 1.7 \\
\hline
\end{tabular}
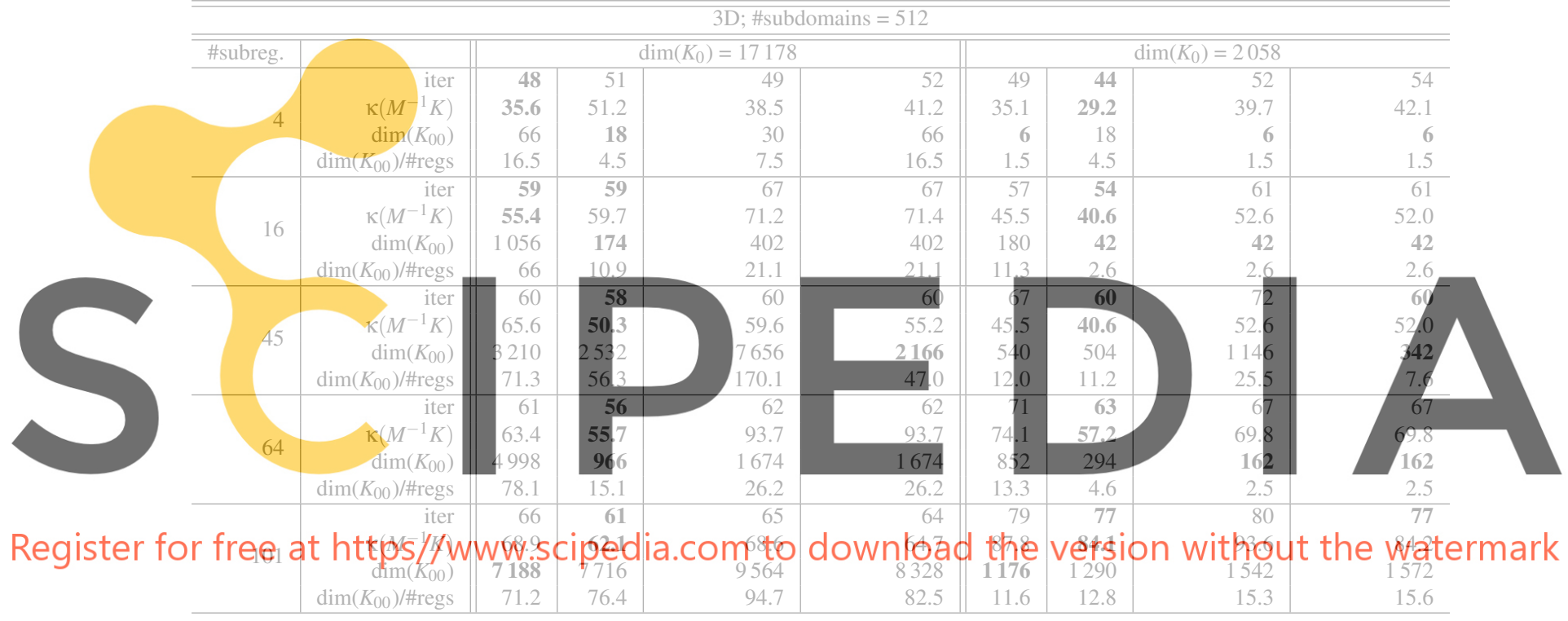

Table 1: Number of PCG iterations Iter, condition number estimate $\kappa\left(M^{-1} K\right)$ and dimension of the coarsest problem $\operatorname{dim}\left(K_{00}\right)$ for the three-level extension with different number of subregions and partitioning methods. The subdomain size is chosen such that each process owns $10^{2}$ respectively $10^{3}$ nodes. We have $\delta=1$ and $\Delta=1$. In each row, the best results for the three-level GDSW and the RGDSW preconditioner are marked in bold.

Robustness The different partitioning methods result in significantly different decompositions into subregions; see Figure 1. This is the case even for a small number of subregions. On the other hand, our algorithm results in similar numbers of iterations and condition number estimates for all partitioning methods. We observe a slight increase in the number of iterations for an increasing number of subregions. The effect is more visible for RGDSW; see Table 1.

Partitioning The different partitioning schemes result in different numbers of interface components, i.e., faces, edges, and vertices. As a result, the size of the coarse matrix $K_{00}$ may differ significantly. In certain cases, we observe unconnected subdregions, which increases the number of interface components 
and therefore increases $\operatorname{dim}\left(K_{00}\right)$; also see Figure 1. In our experiments, ParMETIS sometimes produces a structured decomposition; see the cases of 4 and 16 subregions in Figure 1 . In these cases, $\operatorname{dim}\left(K_{00}\right)$ is small: using 16 subregions instead of 15 subregions in two dimensions reduces $\operatorname{dim}\left(K_{00}\right)$ to about $70 \%$ for ParMETIS as well as for blockwise partitioning; see Table 1. For PHG, we do not see this effect when changing the number of subregions from 15 to 16: the size of $\operatorname{dim}\left(K_{00}\right)$ stays the same for GDSW and reduces only slightly for RGDSW. The results for the test case of 512 subdomains, using 45 and 64 subregions in three dimensions, is consistent with this observation. For 45 subregions, the size of the $K_{00}$ is significantly larger than for 64 subregions using ParMETIS (R), although we would expect the contrary. Here, ParMETIS with partitioning from scratch results in $\operatorname{dim}\left(K_{00}\right)=7656$ for the GDSW coarse space using 45 subregions. This compares to $\operatorname{dim}\left(K_{00}\right)=1674$ for 64 subregions. For the RGDSW coarse space $\operatorname{dim}\left(K_{00}\right)$ decreases by a factor more than 7: we have $\operatorname{dim}\left(K_{00}\right)=1146(45$ subregions) and $\operatorname{dim}\left(K_{00}\right)=162$ (64 subregions); see Table 1 . The PHG and the block approach show more consistent results with respect to $\operatorname{dim}\left(K_{00}\right)$. Note that ParMETIS (R), which uses repartitioning of the linear partition, often yields a smaller $\operatorname{dim}\left(K_{00}\right)$ compared to ParMETIS (P).

In total, the parallel hyper graph (PHG) method from Zoltan, which avoids unconnected subregions, as well as the block-wise approach, avoid outliers with very large $\operatorname{dim}\left(K_{00}\right)$ present in the other methods.

Conclusion Concerning the different graph partitioning method to generate the subregions, our algorithm is robust in the condition number and the number of iterations. However, to keep the cost of the sparse direct linear coarse solver low, we want to keep $\operatorname{dim}\left(K_{00}\right)$ small. For certain special cases, ParMETIS produces a structured decomposition resulting in a very small $\operatorname{dim}\left(K_{00}\right)$. However, changing the numbers of subregions slightly can result in a very large $\operatorname{dim}(K$ titioning a linear decomposition using PaMkTIS (R) can be a better the block-wise partitionin that, for larger problems, block-wise approach as in slower Krylov convergence.

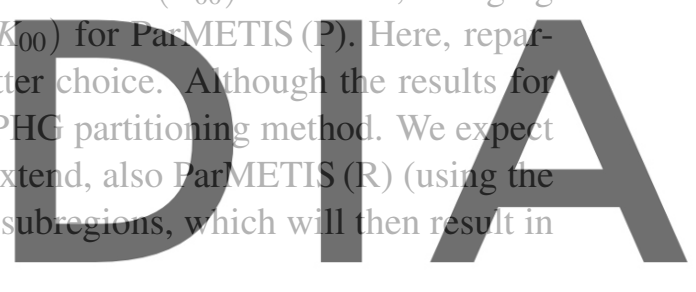

\section{Register for free at https//www.scipedia.com to download the version without the watermark}

The author acknowledge computing time on the Compute Cluster of the Fakultät für Mathematik und İnformatik of Technische Universität Freiberg (DFG project number 397252409), operated by the university computing center (URZ). The second and third author would like to acknowledge funding by the Deutsche Forschungsgemeinschaft (DFG) under the DFG project number 441509557 within the DFG SPP 2256.

\section{REFERENCES}

[1] Trilinos public git repository. Web, 2018. https://github.com/trilinos/trilinos.

[2] S. Badia, A. F. Martín, and J. Principe. Multilevel balancing domain decomposition at extreme scales. SIAM J. Sci. Comput., 38(1):C22-C52, 2016.

[3] Matthias Bollhöfer, Olaf Schenk, Radim Janalik, Steve Hamm, and Kiran Gullapalli. State-of-theArt Sparse Direct Solvers, pages 3-33. Springer International Publishing, Cham, 2020.

[4] C. R. Dohrmann, A. Klawonn, and O. B. Widlund. Domain decomposition for less regular subdomains: overlapping Schwarz in two dimensions. SIAM J. Numer. Anal., 46(4):2153-2168, 2008. 
[5] C. R. Dohrmann, A. Klawonn, and O. B. Widlund. A family of energy minimizing coarse spaces for overlapping Schwarz preconditioners. In Domain decomposition methods in science and engineering XVII, volume 60 of Lect. Notes Comput. Sci. Eng., pages 247-254. Springer, Berlin, 2008.

[6] C. R. Dohrmann and O. B. Widlund. On the design of small coarse spaces for domain decomposition algorithms. SIAM J. Sci. Comput., 39(4):A1466-A1488, 2017.

[7] A. Heinlein, A. Klawonn, S. Rajamanickam, and O. Rheinbach. FROSch: A Fast And Robust Overlapping Schwarz Domain Decomposition Preconditioner Based on Xpetra in Trilinos, pages 176-184. Springer, 2020. Preprint https://kups.ub.uni-koeln. de/9018/.

[8] A. Heinlein, A. Klawonn, O. Rheinbach, and F. Röver. A three-level extension for fast and robust overlapping schwarz (FROSch) preconditioners with reduced dimensional coarse space. In preparation.

[9] A. Heinlein, A. Klawonn, O. Rheinbach, and F. Röver. A three-level extension of the GDSW overlapping Schwarz preconditioner in two dimensions. In T. Apel, U. Langer, A. Meyer, and O. Steinbach, editors, Advanced Finite Element Methods with Applications: Selected Papers from the 30th Chemnitz Finite Element Symposium 2017, pages 187-204. Springer, Cham, 2019.

[10] A. Heinlein, A. Klawonn, O. Rheinbach, and F. Röver. A three-level extension of the GDSW overlapping Schwarz preconditioner in three dimensions. In R. Haynes, S. MacLachlan, X. Cai, L. Halpern, H. H. Kim, A. Klawonn, and O. Widlund, editors, Domain Decomposition Methods in Science and Engineering XXV, pages 185-192, Cham, 2020. Springer International Publishing.
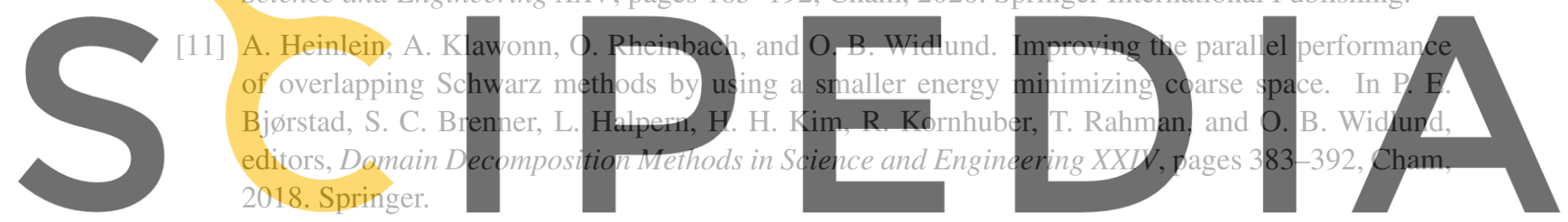

[12] Pierre Jolivet, Frédéric Hecht, Frédéric Nataf, and Christophe Prud'homme. Scalable domain de-

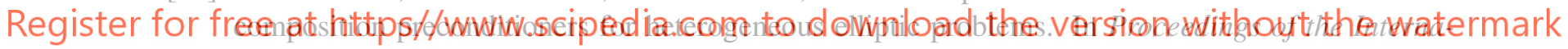
tional Conference on High Performance Computing, Networking, Storage and Analysis, SC '13, pages 80:1-80:11, New York, NY, USA, 2013. ACM.

[13] G. Karypis and K. Schloegel. ParMETIS-parallel graph partitioning and sparse matrix ordering library version 4.0. Technical report, University of Minnesota, Department of Computer Science and Engineering Minneapolis, March 2013.

[14] J. Mandel, B. Sousedík, and C. R. Dohrmann. Multispace and multilevel BDDC. Computing, 83(2-3):55-85, 2008.

[15] J. Sístek, B. Sousedík, J. Mandel, and P. Burda. Parallel implementation of multilevel BDDC. In Numerical mathematics and advanced applications 2011, 2011. 9th European conference on numerical mathematics and advanced applications, Leicester, UK, September 59, 2011.

[16] X. Tu. Three-level BDDC in two dimensions. International Journal for Numerical Methods in Engineering, 69(1):33-59, 2007.

[17] The Zoltan2 Project Team. The Zoltan2 Project Website. 